\title{
Universiteit
}

Leiden

The Netherlands

\section{Signature of Fermi-surface anisotropy in point contact conductance in the presence of defects}

Avotina, Y.S.; Kolesnichenko, Y.A.; Otte, A.F.; Ruitenbeek, J.M. van

\section{Citation}

Avotina, Y. S., Kolesnichenko, Y. A., Otte, A. F., \& Ruitenbeek, J. M. van. (2006). Signature of Fermi-surface anisotropy in point contact conductance in the presence of defects. Physical Review B, 74, 085411. doi:10.1103/PhysRevB.74.085411

Version: $\quad$ Not Applicable (or Unknown)

License: $\quad$ Leiden University Non-exclusive license

Downloaded from: https://hdl.handle.net/1887/62234

Note: To cite this publication please use the final published version (if applicable). 


\title{
Signature of Fermi-surface anisotropy in point contact conductance in the presence of defects
}

\author{
Ye. S. Avotina, ${ }^{1,2}$ Yu. A. Kolesnichenko, ${ }^{1,2}$ A. F. Otte, ${ }^{2}$ and J. M. van Ruitenbeek ${ }^{2}$ \\ ${ }^{1}$ B.I. Verkin Institute for Low Temperature Physics and Engineering, National Academy of Sciences of Ukraine, 47, Lenin Ave., \\ 61103, Kharkov, Ukraine \\ ${ }^{2}$ Kamerlingh Onnes Laboratorium, Universiteit Leiden, Postbus 9504, 2300 Leiden, The Netherlands
}

(Received 3 April 2006; published 16 August 2006)

\begin{abstract}
In a previous paper [Avotina et al., Phys. Rev. B 71, 115430 (2005)] we have shown that in principle it is possible to image the defect positions below a metal surface by means of a scanning tunneling microscope. The principle relies on the interference of electron waves scattered on the defects, which give rise to small but measurable conductance fluctuations. Whereas in that work the band structure was assumed to be free-electron like, here we investigate the effects of Fermi surface anisotropy. We demonstrate that the amplitude and period of the conductance oscillations are determined by the local geometry of the Fermi surface. The signal results from those points for which the electron velocity is directed along the vector connecting the point contact to the defect. For a general Fermi surface geometry the position of the maximum amplitude of the conductance oscillations is not found for the tip directly above the defect. We have determined optimal conditions for determination of defect positions in metals with closed and open Fermi surfaces.
\end{abstract}

DOI: 10.1103/PhysRevB.74.085411

PACS number(s): 73.23.-b, 72.10.Fk

\section{INTRODUCTION}

The interference of electron waves scattered by single defects results in an oscillatory dependence of the point contact conductance $G(V)$ on the applied voltage $V$. This effect originates from quantum interference between the principal wave that is directly transmitted through the contact and the partial wave that is scattered by the contact and the defect or several defects. Such conductance oscillations have been observed in quantum point contacts ${ }^{1-4}$ and investigated theoretically in the papers. ${ }^{3,5-7}$

In our previous paper $^{8}$ the oscillatory voltage dependence of the conductance of a tunnel point contact in the presence of a single pointlike defect has been analyzed theoretically and it has been shown that this dependence can be used for the determination of defect positions below a metal surface by means of a scanning tunneling microscope (STM). In the model of a spherical Fermi surface (FS) the amplitude of the conductance oscillations is maximal when the contact is placed directly above the defect. The oscillatory part of the conductance $\Delta G$ for this situation is proportional to

$$
\Delta G(V) \sim \sin \left(2 z_{0} \sqrt{k_{F}^{2}+\frac{2 m e V}{\hbar^{2}}}\right),
$$

where $z_{0}$ the depth of the defect and $k_{F}$ and $m$ are the Fermi wave vector and effective mass of the electrons. ${ }^{8}$ Materials with an almost spherical FS are most suitable for this model.

In most metals the dispersion relation for the charge carriers is a complicated anisotropic function of the momentum. This leads to anisotropy of the various kinetic characteristics. ${ }^{9}$ Particularly, as shown in Ref. 10, the current spreading may be strongly anisotropic in the vicinity of a point contact. This effect influences the way the point contact conductance depends on the position of the defect. For example, in the case of a $\mathrm{Au}(111)$ surface the necks in the FS should cause a defect to be invisible when probed exactly from above.
Qualitatively, the wave function of electrons injected by a point contact for arbitrary FS $\varepsilon(\mathbf{p})=\varepsilon_{F}$ has been analyzed by Kosevich. ${ }^{10} \mathrm{He}$ noted that at large distances from the contact the electron wave function for a certain direction $\mathbf{r}$ is defined by those points on the FS for which the electron group velocity is parallel to $\mathbf{r}$. Unless the entire FS is convex there are several such points. The amplitude of the wave function depends on the Gaussian curvature $K$ in these points, which can be convex $(K>0)$ or concave $(K<0)$. The parts of the FS having different signs of curvature are separated by lines of $K=0$ (inflection lines). In general there is a continuous set of electron wave vectors for which $K=0$. The electron flux in the directions having zero Gaussian curvature exceeds the flux in other directions. ${ }^{10}$

Electron scattering by defects in metals with an arbitrary FS can be strongly anisotropic. ${ }^{9}$ Generally, the wave function of the electrons scattered by the defect consists of several superimposed waves, which travel with different velocities. In the case of an open constant-energy surface there are directions along which the electrons cannot move at all. Scattering events along those directions occur only if the electron is transferred to a different sheet of the FS. ${ }^{9}$

In this paper we analyze the effect of anisotropy of the FS to the possibility of determination of the position of a defect below a metal surface by use of a STM. We show that the amplitude and the period of the conductance oscillations are defined by the local geometry of the FS, namely by those points for which the electron group velocity is directed along the radius vector from the contact to the defect. General formulas for the wave function and point contact conductance are obtained in Secs. II and III. In Sec. IV the asymptotic forms of the wave function and the point contact conductance for large distances of the defect from the contact are found. The general results are illustrated for two specific models of the FS: an ellipsoid (Sec. V) and a corrugated cylinder (Sec. VI). Using these models, for which analytical dependencies of the conductance on voltage and defect position can be found, we describe the manifestation of com- 
mon features of FS geometries to the conductance oscillations: anisotropy of a convex part (bellies), changing of the curvature (inflection lines) and presence of open directions (necks).

\section{THE SCHRÖDINGER EQUATION FOR QUASIPARTICLES}

Let us consider as a model for our system a nontransparent interface located at $z=0$ separating two metal half-spaces, in which there is an orifice (contact) of radius $R$ centered at the point $\mathbf{r}=0$. The potential barrier in the plane $z=0$ is taken to be a delta function,

$$
U(\mathbf{r})=U f(\boldsymbol{\rho}) \delta(z),
$$

where $\boldsymbol{\rho}=(x, y)$ is a two-dimensional vector in the plane of the interface, with $\mathbf{r}=(\boldsymbol{\rho}, z)$. The function $f(\boldsymbol{\rho}) \rightarrow \infty$ in all points of the plane $z=0$ except in the contact, where $f(\boldsymbol{\rho})$ $=1$. At the point $\mathbf{r}=\mathbf{r}_{0}$ near the contact in the upper halfspace, $z>0$, a pointlike defect is placed. The electron interaction with the defect is described by the potential $D(\mathbf{r}$ $-\mathbf{r}_{0}$ ), which is confined to a small region with a characteristic radius $r_{D}$ around the point $\mathbf{r}_{0}$.

It is known that one can obtain an effective Schrödinger equation for quasiparticles in a metal from the dispersion relation $\varepsilon(\mathbf{p})$ (the band structure) by replacement of the quasimomentum $\mathbf{p}$ (below for short we write momentum) in the function $\varepsilon(\mathbf{p})$ with the momentum operator $\hat{\mathbf{p}}=\frac{\hbar}{i} \nabla .{ }^{9}$ Here we do not specify the specific form of the dependence $\varepsilon(\mathbf{p})$, except that it satisfies the general condition of point symmetry $\varepsilon(\mathbf{p})=\varepsilon(-\mathbf{p})$. For simplicity we assume that FS has only one sheet; there is only one zone described by the function $\varepsilon(\mathbf{p})$. In the reduced zone scheme a given momentum $\mathbf{p}$ identifies a single point within the first Brillouin zone. The wave function $\psi(\mathbf{r})$ satisfies the Schrödinger equation with an effective Hamiltonian $\varepsilon(\hat{\mathbf{p}})$,

$$
\varepsilon(\hat{\mathbf{p}}) \psi(\mathbf{r})+[\varepsilon-U(\mathbf{r})-e V(z)] \psi(\mathbf{r})=D\left(\mathbf{r}-\mathbf{r}_{0}\right) \psi(\mathbf{r}),
$$

where $U(\mathbf{r})$ is defined by Eq. (1), $V(z)$ is the applied electrical potential, and $\varepsilon$ is the electron energy.

We consider a large barrier potential $U$. In this case the amplitude $t$ of the electron wave function passing through the barrier is

$$
t\left(\varepsilon, \mathbf{p}_{t}\right) \approx \frac{\hbar\left(v_{z}^{\mathrm{in}}-v_{z}^{\mathrm{ref}}\right)}{2 i U},
$$

where $v_{z}^{\text {in }}$ and $v_{z}^{\text {ref }}$ are the $z$ components of the velocity $\mathbf{v}$ $=\partial \varepsilon(\mathbf{p}) / \partial \mathbf{p}$ of incident electrons (in) and electrons specularly reflected by the barrier (ref), respectively. Under condition of specular reflection the energy $\varepsilon$ and the component of the momentum tangential to the interface, $\mathbf{p}_{t}=\left(p_{x}, p_{y}\right)$ at $z=0$ are conserved. The components of the electron momentum perpendicular to the interface, $p_{z}^{\text {in }}\left(\mathbf{p}_{t}, \varepsilon\right)$ and $p_{z}^{\text {ref }}\left(\mathbf{p}_{t}, \varepsilon\right)$ are related by the equations,

$$
\varepsilon\left(\mathbf{p}_{t}^{\text {in }}, p_{z}^{\text {in }}\right)=\varepsilon\left(\mathbf{p}_{t}^{\text {ref }}, p_{z}^{\text {ref }}\right)=\varepsilon, \quad \mathbf{p}_{t}^{\text {in }}=\mathbf{p}_{t}^{\text {ref }} \equiv \mathbf{p}_{t} .
$$

The velocities $v_{z}^{\text {in }}$ and $v_{z}^{\text {ref }}$ have the opposite sign

$$
\mathbf{v}^{\text {in }} \cdot \mathbf{N}<0, \quad \mathbf{v}^{\text {ref }} \cdot \mathbf{N}>0,
$$

where $\mathbf{N}$ is a unit vector normal to the interface laying in the half-space of the electron wave under consideration $[\mathbf{N}$ $=(0,0,1)$ for $z>0$ and $\mathbf{N}=(0,0,-1)$ for $z<0]$. We will assume that the crystallographic axes in half-spaces $z \gtrless 0$ are identical. In this case the momenta and velocities for electrons incident on the barrier and for those transmitted through the barrier are equal.

In general Eq. (4) may have several solutions, i.e., several specularly reflected states may correspond to an incident state with momentum $p_{z}^{\text {in }}$. Such reflection is called multichannel specular reflection. ${ }^{11}$ Below we assume that there is only one reflected electron state.

In the limit of a small probability of electron tunneling through the barrier, $|t|^{2} \ll 1$ the applied voltage drops entirely over the barrier and we take the electric potential to be a step function $V(z)=V \Theta(-z)$. The reference point of zero electron energy is the bottom of the conduction band in the upper half-space, $z>0$. The conduction band in the lower halfspace $z<0$ is shifted by a value $e V$. We also assume that the applied bias $\mathrm{eV}$ is much smaller than the Fermi energy and in solving the Schrödinger Eq. (2) we neglect the electric potential $V(z)$. Equation (2) can be solved by using perturbation theory with the small parameter $|t| \ll 1 .^{12}$ In the zeroth approximation in this parameter we have the problem of an impenetrable partition between two metal half-spaces.

We start by solving for the wave function $\psi^{(0)}\left(\varepsilon, \mathbf{p}_{t} ; \mathbf{r}\right)$ for a tunneling point contact of low transparency, $|t| \ll 1$ without defects $\left[D\left(\mathbf{r}-\mathbf{r}_{0}\right)=0\right]$. The wave function $\psi_{0}^{(0)}(\mathbf{r})$, in zeroth order in the parameter $|t| \ll 1$, satisfies the boundary condition $\psi_{0}^{(0)}(z=0)=0$ at the interface,

$$
\psi_{0}^{(0)}\left(\varepsilon, \mathbf{p}_{t} ; \mathbf{r}\right)=e^{i \mathbf{p}_{t} \rho / \hbar}\left(e^{i p_{z}^{\mathrm{in}} z / \hbar}-e^{i p_{z}^{\mathrm{ref}} z / \hbar}\right) .
$$

Let us consider an electron wave $\exp (i \mathbf{p r} / \hbar)$ incident on the junction from the lower half-space $z<0$, so that $v_{z}>0$. In this half-space to first approximation in the parameter $t$ the solution $\psi^{(0)}(\mathbf{r})$ of the homogeneous Schrödinger equation can be written in the form ${ }^{12}$

$$
\psi^{(0)}(\mathbf{r})=\psi_{0}^{(0)}(\mathbf{r})+\varphi^{(-)}(\mathbf{r}), \quad z<0,
$$

where the second term, $\varphi^{(-)}(\mathbf{r}) \propto t$, describes the changes in the reflected wave as a result of transmission through the contact. The wave function transmitted into the half-space $z>0$ is proportional to the amplitude $t$,

$$
\psi^{(0)}(\mathbf{r})=\varphi^{(+)}(\mathbf{r}), \quad z>0 .
$$

The function $\psi^{(0)}(\boldsymbol{\rho}, z)$ satisfies the condition of continuity and the condition of conservation of probability flow at $z$ $=0$. For small $|t|$ these conditions reduce to

$$
\begin{gathered}
\varphi^{(-)}(\boldsymbol{\rho}, 0)=\varphi^{(+)}(\boldsymbol{\rho}, 0), \\
t e^{i \mathbf{p}_{\mathbf{p}} \boldsymbol{\rho} / \hbar}=f(\boldsymbol{\rho}) \varphi^{(+)}(\boldsymbol{\rho}, 0) .
\end{gathered}
$$

In the absence of defects $(D=0)$ the solution of the Schrödinger equation is given by ${ }^{8,12}$ 


$$
\varphi^{(+)}(\boldsymbol{\rho}, z)=t \int_{-\infty}^{\infty} \frac{d \mathbf{p}_{t}^{\prime}}{(2 \pi \hbar)^{2}} F\left(\mathbf{p}_{t}-\mathbf{p}_{t}^{\prime}\right) e^{i\left[\mathbf{p}_{t}^{\prime} \boldsymbol{\rho}+p_{z}^{(+)}\left(\varepsilon, \mathbf{p}_{t}^{\prime}\right) z\right] / \hbar},
$$

where

$$
F\left(\mathbf{p}_{t}-\mathbf{p}_{t}^{\prime}\right)=\int_{-\infty}^{\infty} d \boldsymbol{\rho} \frac{e^{i\left(\mathbf{p}_{t}-\mathbf{p}_{t}^{\prime}\right) \boldsymbol{\rho} / \hbar}}{f(\boldsymbol{\rho})}
$$

$p_{z}^{( \pm)}\left(\varepsilon, \mathbf{p}_{t}^{\prime}\right)$ are roots of the equation

$$
\varepsilon\left(\mathbf{p}_{t}^{\prime}, p_{z}^{( \pm)}\right)=\varepsilon(\mathbf{p}),
$$

corresponding to waves with velocities $v_{z}^{(+)}\left(\varepsilon, \mathbf{p}_{t}^{\prime}\right)>0$ and $v_{z}^{(-)}\left(\varepsilon, \mathbf{p}_{t}^{\prime}\right)<0$.

Let $D(\mathbf{r})$ be a spherically symmetric scattering potential for a pointlike defect, with a range $r_{D}$ that is order of the Fermi wavelength $\lambda_{F}[D \ll D(0)$, the maximal value of $D$, when $\left.\left|\mathbf{r}-\mathbf{r}_{0}\right| \gg r_{D}\right]$. For a pointlike defect $\left(r_{D} \rightarrow 0\right)$, the righthand side in the Schrödinger Eq. (2) can be rewritten as $D\left(\mathbf{r}-\mathbf{r}_{0}\right) \psi\left(\mathbf{r}_{0}\right) .{ }^{13}$ This makes it possible to find a solution to Eq. (2) by means of the Green function $G_{0}^{+}\left(\mathbf{r}^{\prime}, \mathbf{r} ; \varepsilon\right)$ of the homogeneous equation (at $D=0$ ). The wave function scattered from the defect, $\psi(\mathbf{r})$, can be expressed in terms of the wave function $\varphi^{(+)}(\mathbf{r})$ transmitted into the upper metal halfspace,

$$
\psi(\mathbf{r})=\varphi^{(+)}(\mathbf{r})+\varphi^{(+)}\left(\mathbf{r}_{0}\right) \frac{J\left(\mathbf{r}, \mathbf{r}_{0}\right)}{1-J\left(\mathbf{r}_{0}, \mathbf{r}_{0}\right)}
$$

where

$$
J\left(\mathbf{r}, \mathbf{r}_{0}\right)=\int d \mathbf{r}^{\prime} D\left(\mathbf{r}^{\prime}-\mathbf{r}_{0}\right) G_{0}^{+}\left(\mathbf{r}^{\prime}, \mathbf{r} ; \varepsilon\right) .
$$

Because the Green function has a singularity at $\mathbf{r} \rightarrow \mathbf{r}^{\prime}$, Eq. (14) is correct if the integral $J\left(\mathbf{r}, \mathbf{r}_{0}\right)$ (15) converges in the point $\mathbf{r}=\mathbf{r}_{0}$.

To proceed with further calculations we assume that the scattering potential is small and use perturbation theory in the interaction with the defect. This implies that we take $\left|J\left(\mathbf{r}_{0}, \mathbf{r}_{0}\right)\right| \ll 1$. The wave function solution, that is linear in $|t|$ and with a contribution to first order in $D$, is

$$
\psi(\mathbf{r})=\varphi^{(+)}(\mathbf{r})+\varphi^{(+)}\left(\mathbf{r}_{0}\right) \int d \mathbf{r}^{\prime} D\left(\mathbf{r}^{\prime}-\mathbf{r}_{0}\right) G_{0}^{+}\left(\mathbf{r}, \mathbf{r}^{\prime} ; \varepsilon\right) .
$$

The Green function $G_{0}\left(\mathbf{r}, \mathbf{r}^{\prime} ; \varepsilon\right)$ in zeroth approximation in the parameters $D$ and $|t|$ should be calculated from the wave functions (6),

$$
G_{0}^{+}\left(\mathbf{r}, \mathbf{r}^{\prime} ; \varepsilon\right)=\int \frac{d \mathbf{p}}{(2 \pi \hbar)^{3}} \frac{\psi_{0}^{(0)}(\mathbf{r})\left[\psi_{0}^{(0)}\left(\mathbf{r}^{\prime}\right)\right]^{*}}{\varepsilon-\varepsilon(\mathbf{p})-i 0}
$$

This is the Green function of the Schrödinger equation in the half-space $z>0$ with hard-wall boundary conditions at $z=0$. Substituting the wave function $\psi_{0}^{(0)}(\mathbf{r})$, Eq. (6), for $z>0$ in Eq. (17), we find

$$
\begin{aligned}
G_{0}^{+}\left(\mathbf{r}, \mathbf{r}^{\prime} ; \varepsilon\right)= & \frac{1}{i \hbar} \int \frac{d \mathbf{p}_{t}}{(2 \pi \hbar)^{2}} \frac{e^{i \mathbf{p}_{t}\left(\boldsymbol{\rho}-\boldsymbol{\rho}^{\prime}\right) / \hbar+i p_{z}^{(+)} z / \hbar}}{v_{z}^{(+)}-v_{z}^{(-)}}\left(e^{-i p_{z}^{(+)} z^{\prime} / \hbar}\right. \\
& \left.-e^{-i p_{z}^{(-)} z^{\prime} / \hbar}\right), \quad z>z^{\prime} .
\end{aligned}
$$

For $0<z<z^{\prime}$ one should make the replacements $z \leftrightarrow z^{\prime}$ and $p_{z}^{(-)} \leftrightarrow-p_{z}^{(+)}$in Eq. (18); $p_{z}^{( \pm)}$are given by Eq. (13).

The main contribution to the integral in Eq. (16) comes from a small region near the point $\mathbf{r}^{\prime}=\mathbf{r}_{0}$. Far from the point $\mathbf{r}=\mathbf{r}_{0}\left(\left|\mathbf{r}-\mathbf{r}_{0}\right| \gg r_{D}\right)$ the solution (16) takes the form

$$
\psi(\mathbf{r})=\varphi^{(+)}(\mathbf{r})+g G_{0}^{+}\left(\mathbf{r}, \mathbf{r}_{0} ; \varepsilon\right) \varphi^{(+)}\left(\mathbf{r}_{0}\right),
$$

where

$$
g=\int d \mathbf{r}^{\prime} D\left(\mathbf{r}^{\prime}-\mathbf{r}_{0}\right)
$$

is the constant of electron-impurity interaction.

\section{POINT-CONTACT CONDUCTANCE}

The electrical current $I(V)$ can be evaluated from the electron wave functions, $\psi$, of the system through ${ }^{14}$

$$
I(V)=\frac{2 e}{(2 \pi \hbar)^{3}} \int d \mathbf{p} I_{\mathbf{p}} \Theta\left(v_{z}\right)\left[n_{F}(\varepsilon)-n_{F}(\varepsilon+e V)\right] .
$$

Here

$$
I_{\mathbf{p}}=\int_{-\infty}^{\infty} d x d y \operatorname{Re}\left(\psi^{*} \hat{v}_{z} \psi\right)
$$

is the density of probability flow in the $z$ direction for the momentum $\mathbf{p}$, integrated over a plane $z=x$ const, $n_{F}(\varepsilon)$ is the Fermi distribution function, and $\hat{\mathbf{v}}$ is the velocity operator, $\hat{\mathbf{v}}=\partial \varepsilon(\hat{\mathbf{p}}) / \partial \hat{\mathbf{p}}$. For the definiteness we choose in Eq. (21) $\mathrm{eV}$ $>0$. At low temperatures the tunnel current is due to those electrons in the half-space $z<0$ having an energy between the Fermi energy, $\varepsilon_{F}$, and $\varepsilon_{F}+e V$, because on the other side of the barrier $z>0$ only states with $\varepsilon \geqslant \varepsilon_{F}$ are available.

After performing the integration over a plane at $z \gg z_{0}$, where the wave function (19) can be used, we find the density flow (22) becomes

$$
\begin{aligned}
I_{\mathbf{p}}= & \left|t\left(\varepsilon, \mathbf{p}_{t}\right)\right|^{2} \pi^{3} \hbar R^{4}\left\langle\left(v_{z}^{(+)}\right)^{2}\right\rangle_{\varepsilon} \nu(\varepsilon) \\
& +\frac{\left|t\left(\varepsilon, \mathbf{p}_{t}\right)\right|^{2} g \pi^{2} R^{4}}{\hbar} \operatorname{Re} \int \frac{d \mathbf{p}_{t}^{\prime}}{(2 \pi \hbar)^{2}} \frac{i v_{z}^{(+)}\left(\mathbf{p}_{t}^{\prime}\right) e^{-i \mathbf{p}_{t}^{\prime} \boldsymbol{\rho}_{0} / \hbar}}{v_{z}^{(+)}\left(\mathbf{p}_{t}^{\prime}\right)-v_{z}^{(-)}\left(\mathbf{p}_{t}^{\prime}\right)} \\
& \times\left(e^{-i p_{z}^{(+)}\left(\mathbf{p}_{t}^{\prime}\right) z_{0} / \hbar}-e^{-i p_{z}^{(-)}\left(\mathbf{p}_{t}^{\prime}\right) z_{0} / \hbar}\right) \\
& \times \int_{-\infty}^{\infty} \frac{d \mathbf{p}_{t}^{\prime \prime}}{(2 \pi \hbar)^{2}} e^{i\left[\mathbf{p}_{t}^{\prime \prime} \boldsymbol{\rho}_{0}+p_{z}^{(+)}\left(\mathbf{p}_{t}^{\prime \prime}\right) z_{0}\right] / \hbar}
\end{aligned}
$$

where the mean value at energy $\varepsilon$ is obtained by integration over the surfaces of constant energy in momentum space, 


$$
\langle\cdots\rangle_{\varepsilon}=\frac{\int_{\varepsilon_{\mathbf{p}}=\varepsilon} \frac{d S_{\mathbf{p}}}{|\mathbf{v}|} \ldots}{\int_{\varepsilon_{\mathbf{p}}=\varepsilon} \frac{d S_{\mathbf{p}}}{|\mathbf{v}|}},
$$

scaled by the velocity, $|\mathbf{v}|=\left|\frac{\partial \varepsilon}{\partial \mathbf{p}}\right|$. In Eq. (23), $p_{z}^{(+)}\left(\mathbf{p}_{t}\right)$ and $p_{z}^{(-)}\left(\mathbf{p}_{t}\right)$ are given by Eq. (13) and $\nu(\varepsilon)$ is the electron density of states per unit volume.

Taking into account Eq. (23) we can calculate the currentvoltage characteristics $I(V)$. The conductance $G(V)$ is the first derivative of the current $I(V)$ with $V$ and in the limit $T$ $\rightarrow 0$ we obtain

$$
G(V)=\frac{\partial I}{\partial V}=e^{2} \nu(\varepsilon)\left\langle I_{\mathbf{p}}\right\rangle_{\varepsilon_{F}+e V} .
$$

After integration over $S_{\mathbf{p}}$ (24), Eq. (25) should be expanded in the parameter $e V / \varepsilon_{F} \ll 1$.

\section{ASYMPTOTICS OF THE WAVE FUNCTION AND THE CONDUCTANCE}

In this section we find the wave function at large distances from the contact, $r \gg \lambda_{F}$, and an asymptotic expression for the conductance in the limit of a large distance between the defect and the contact, $r_{0} \gg \lambda_{F}$ and a small contact radius, $R \ll \lambda_{F}$, where $\lambda_{F}$ is the characteristic electron Fermi wavelength. For $R \rightarrow 0$ the function $F$ in Eq. (12) takes the form ${ }^{8}$

$$
F\left(\mathbf{p}_{t}-\mathbf{p}_{t}^{\prime}\right)=\pi R^{2},
$$

and the wave function (11) can be written as

$$
\varphi^{(+)}(\boldsymbol{\rho}, z)=t\left(\varepsilon, \mathbf{p}_{t}\right) \pi R^{2} \int_{-\infty}^{\infty} \frac{d \mathbf{p}_{t}^{\prime}}{(2 \pi \hbar)^{2}} e^{i\left[\mathbf{p}_{t}^{\prime} \boldsymbol{\rho}+p_{z}^{(+)}\left(\mathbf{p}_{t}^{\prime}\right) z\right] / \hbar}
$$

Let us consider the integral

$$
\Lambda(\mathbf{r}, \varepsilon)=\int_{-\infty}^{\infty} \frac{d \mathbf{p}_{t}^{\prime}}{(2 \pi \hbar)^{2}} e^{i \Gamma\left(\mathbf{p}_{t}, \mathbf{r}\right)},
$$

where $\Gamma\left(\mathbf{p}_{t}, \mathbf{r}\right)$ is the phase accumulated over the path traveled by the electron between the contact and the point $\mathbf{r}$,

$$
\Gamma\left(\mathbf{p}_{t}, \mathbf{r}\right)=\frac{1}{\hbar}\left[\mathbf{p}_{t} \boldsymbol{\rho}+p_{z}^{(+)}\left(\mathbf{p}_{t}\right) z\right] .
$$

This kind of integral appears in the expressions for the wave function [Eqs. (27), (18), and (19)] and for the conductance [Eqs. (23) and (25)]. At a large distance, $r \gg \lambda_{F}$, the exponent under the integral in Eq. (28) is a rapidly oscillating function and the integral can be calculated by the stationary phase method (see, for example, Ref. 15). The stationary phase points $\mathbf{p}_{t}=\mathbf{p}_{t}^{\text {(st) }}$ are defined by the equation

$$
\left.\frac{\partial \Gamma}{\partial \mathbf{p}_{t}}\right|_{\mathbf{p}_{t}=\mathbf{p}_{t}^{(\mathrm{st})}}=0 .
$$

With Eq. (29) we find

$$
\boldsymbol{\rho}+\left.z \frac{\partial p_{z}^{(+)}\left(\mathbf{p}_{t}\right)}{\partial \mathbf{p}_{t}}\right|_{\mathbf{p}_{t}=\mathbf{p}_{t}^{(\mathrm{st})}}=\boldsymbol{\rho}-\left.z \frac{\mathbf{v}_{t}^{(+)}\left(\mathbf{p}_{t}\right)}{v_{z}\left(\mathbf{p}_{t}\right)}\right|_{\mathbf{p}_{t}=\mathbf{p}_{t}^{(\mathrm{st})}}=0 .
$$

For $r \gg \lambda_{F}$ the asymptotic value $\Lambda^{\text {as }}(\mathbf{r}, \varepsilon)$ of the integral (28) is given by

$$
\begin{aligned}
\Lambda^{\mathrm{as}}(\mathbf{r}, \varepsilon)= & \frac{\cos \vartheta}{2 \pi \hbar r \sqrt{\left|K_{0}\right|}} \exp \left[i \Gamma_{0}+i \frac{\pi}{4} \operatorname{sgn}\left(\frac{\partial^{2} p_{z}}{\partial p_{x}^{(\mathrm{st})}}\right)(1\right. \\
& \left.\left.+\operatorname{sgn} K_{0}\right)\right] .
\end{aligned}
$$

Here

$$
\Gamma_{0}(\varepsilon, \mathbf{r})=\Gamma\left(\mathbf{p}_{t}^{(\mathrm{st})}, \mathbf{r}\right)
$$

is the phase (29) in a point defined by Eq. $(31), K(\varepsilon, \mathbf{p})$ is the Gaussian curvature of the surface of constant energy $\varepsilon\left(p_{x}, p_{y}, p_{z}\right)=\varepsilon$, and $\cos \vartheta(\mathbf{r})=z / r$ is the angle between the vector $\mathbf{r}$ and the $z$ axis. At the stationary phase points the curvature $K(\varepsilon, \mathbf{p})$ can be written as

$$
K_{0}(\varepsilon, \mathbf{n})=\left(\frac{1}{|\mathbf{v}|^{2}} \sum_{i, k=x, y, z} A_{i k} n_{i} n_{k}\right)_{\mathbf{p}_{t}=\mathbf{p}_{t}^{(\mathrm{st})}},
$$

where $A_{i k}=\frac{\partial \operatorname{det}\left(\mathbf{m}^{-1}\right)}{\partial m_{i k}^{-1}(\mathbf{p})}$ is the algebraic adjunct of the element

$$
m_{i k}^{-1}(\mathbf{p})=\frac{\partial^{2} \varepsilon}{\partial p_{i} \partial p_{k}}
$$

of the inverse mass matrix $\mathbf{m}^{-1},{ }^{16} n_{i}$ are components of the unit vector $\mathbf{n}=\mathbf{r} / r$. Note that for an arbitrary FS $m_{i k}(\mathbf{p})$ in the point $\mathbf{p}_{t}=\mathbf{p}_{t}^{(\text {st) }}$ depends on the direction of vector $\mathbf{r}$. It follows from Eq. (31) that the velocity at the stationary phase point $\mathbf{p}_{t}^{\text {(st) }}$ is parallel to the radius vector $\mathbf{r}=(\boldsymbol{\rho}, z)$.

If the curvature of the FS changes sign, Eq. (31) has more than one solution $\mathbf{p}_{t}=\mathbf{p}_{t, s}^{(\mathrm{st})}(s=1,2, \ldots)$. In that case the value of the integral (28) is replaced by a sum over all points $\mathbf{p}_{t, s}^{\text {(st) }}$, which in the limit of large distances is

$$
\Lambda(\mathbf{r}, \varepsilon) \approx \sum_{s} \Lambda_{s}^{\mathrm{as}}(\mathbf{r}, \varepsilon),
$$

with $\Lambda_{s}^{\text {as }}(\mathbf{r}, \varepsilon)$ given by Eq. (32) for each stationary phase point $s$. It may also occur that Eq. (31) does not have any solution for given directions of the vector $\mathbf{r}$, and the electron cannot propagate along these directions. These two energy surface properties result in complicated patterns of the distribution of the modulus of the wave function: (1) For directions for which Eq. (31) has several solutions a quantum interference pattern of the electron waves with different velocities should be observed. (2) When Eq. (31) has no solution for the selected direction of the vector $\mathbf{r}$ classical motion in this direction is forbidden and the wave function is exponentially small.

For large values $r, r^{\prime} \gg \lambda_{F}$ the asymptotic behaviors of the Green function, Eq. (18), and of the conductance, Eq. (25) at $r_{0} \gg \lambda_{F}$, can be found analogous to the evaluation of the integral in Eq. (28). The slowly varying functions of the mo- 
mentum must be taken in the stationary phase point. For the partial wave scattered by the defect that is moving towards the interface $p_{z}^{(+)}\left(\mathbf{p}_{t}\right)$ in Eq. (29) must be replaced by $p_{z}^{(-)}\left(\mathbf{p}_{t}\right)$. In this case the stationary points have a group velocity $\mathbf{v}^{(-)}$directed from the point $\mathbf{r}_{0}$ towards the contact. From the central symmetry of the FS, $\varepsilon(\mathbf{p})=\varepsilon(-\mathbf{p})$, it follows that the two stationary phase points for the function $\Gamma\left(\mathbf{p}_{t}, \mathbf{r}\right)$ are antiparallel, $\mathbf{p}^{(+)(\mathrm{st})}=-\mathbf{p}^{(-)(\mathrm{st})} \equiv \mathbf{p}^{(\mathrm{st})}$.

Next, we derive an asymptotic expression for the wave function (19), with $r,\left|\mathbf{r}-\mathbf{r}_{0}\right| \gg \lambda_{F}$, for a symmetric orientation of the FS with respect to the interface, so that $v_{z}^{(+)}=$ $-v_{z}^{(-)}, p_{z}^{(+)}=-p_{z}^{(-)}$and $m_{i k}^{-1}=0$, if $i \neq k$. Under these conditions the wave function (19) takes the form

$$
\begin{aligned}
\psi(\mathbf{r}) \approx & \varphi^{(+)}(\mathbf{r})+\frac{g i}{4 \pi \hbar} \varphi^{(+)}\left(\mathbf{r}_{0}\right) \sum_{s}\left(\frac { 1 } { v _ { z } ^ { ( + ) } ( \mathbf { p } _ { t , s } ^ { ( 1 ) } ) } \Lambda _ { s } ^ { \mathrm { as } } \left(\boldsymbol{\rho}-\boldsymbol{\rho}_{0}, z\right.\right. \\
& \left.\left.+z_{0} ; \varepsilon\right)-\frac{1}{v_{z}^{(+)}\left(\mathbf{p}_{t, s}^{(2)}\right)} \Lambda_{s}^{\mathrm{as}}\left(\boldsymbol{\rho}-\boldsymbol{\rho}_{0},\left|z-z_{0}\right| ; \varepsilon\right)\right)
\end{aligned}
$$

with

$$
\varphi^{(+)}(\mathbf{r}) \approx t \pi R^{2} \sum_{s} \Lambda_{s}^{\mathrm{as}}(\mathbf{r}, \varepsilon)
$$

In Eq. (37) the velocity $v_{z}^{(+)}$is taken in the stationary phase points $\mathbf{p}_{t}^{(s t)}=\mathbf{p}_{t, s}^{(1,2)}$ corresponding to the directions of the vector with coordinates $\left(\boldsymbol{\rho}-\boldsymbol{\rho}_{0},\left|z \pm z_{0}\right|\right)$.

We have assumed for the Gaussian curvature, Eq. (34), that $K_{0} \neq 0$ in the stationary phase points $\mathbf{p}_{t}^{(\mathrm{st})}$. For those points at which $K_{0}=0$ the integral (28) diverges. This means that the third derivative of the phase $\Gamma\left(\mathbf{p}_{t}, \mathbf{r}\right)$ (29) with respect to $\mathbf{p}_{t}$ must be taken into account. In Sec. V this is done for a model FS having cylindrical symmetry with respect to an open direction. Here we only note that the amplitude of the wave function $\varphi^{(+)}(\mathbf{r})(38)$ in a direction of zero Gaussian curvature is larger than for other directions, and decreases more slowly as compared to the $\sim 1 / r$ dependence of Eq. (32). This results in an enhanced current flow near the cone surface defined by the condition $K_{0}=0 .{ }^{10}$ The same effect also appears in the second term of the wave function (37), that describes the scattered wave: the amplitude of this partial wave is maximal in the directions of zero Gaussian curvature.

If the FS has a flat part, i.e., Eq. (31) holds at all points of a FS region of finite area $S_{\mathrm{fl}}$, the associated electron waves propagate in the metal without decrease of their amplitude. ${ }^{10}$ For the flat part the dispersion relation $\varepsilon(\mathbf{p})$ can be presented as $\varepsilon(\mathbf{p})=\mathbf{v}_{0} \mathbf{p}$, where $\mathbf{v}_{0}=$ const is the electron velocity. For such FS the asymptotic value of the integral (28) is

$$
\Lambda_{\mathrm{fl}}^{\mathrm{as}}(\mathbf{r}, \varepsilon)=\frac{v_{0 z} S_{\mathrm{fl}}}{\left|\mathbf{v}_{0}\right|(2 \pi \hbar)^{2}} \exp \left(\frac{i \varepsilon r}{\hbar\left|\mathbf{v}_{0}\right|}\right) .
$$

When the distance between the contact and the defect is large, $r_{0} \gg \lambda_{F}$, we obtain the conductance of the tunnel junction, using Eq. (23) and the asymptotic expression for the wave function (37),

$$
\begin{aligned}
G= & G_{0}\left(1-\frac{g}{2 \pi \hbar^{2}\left\langle\left(v_{z}^{(+)}\right)^{2}\right\rangle_{\varepsilon_{F}} \nu\left(\varepsilon_{F}\right)}\right. \\
& \times \sum_{s, s^{\prime}} \operatorname{Re} \Lambda_{s}^{\mathrm{as}}\left(\mathbf{r}_{0}, \varepsilon_{F}, e V\right) \\
& \left.\times \operatorname{Im} \Lambda_{s^{\prime}}^{\mathrm{as}}\left(\mathbf{r}_{0}, \varepsilon_{F}, e V\right)\right),
\end{aligned}
$$

where $G_{0}$ is the zero-bias $(e V \rightarrow 0)$ conductance of the junction without defect,

$$
G_{0}=e^{2} \pi^{3} R^{4} \hbar\left\langle|t|^{2}\right\rangle_{\varepsilon_{F}} \nu^{2}\left(\varepsilon_{F}\right)\left\langle\left(v_{z}^{(+)}\right)^{2}\right\rangle_{\varepsilon_{F}} .
$$

In deriving Eq. (40) we have assumed that $e V \ll \varepsilon_{F}$ and $r_{0}$ $\gg \lambda_{F}$. Therefore, all functions of the energy $\varepsilon$ in Eq. (40) can be taken at $\varepsilon=\varepsilon_{F}$, except for the phase $\Gamma_{0}(\varepsilon, \mathbf{r})$. When $e V$ $\ll \varepsilon_{F}$,

$$
\Gamma_{0}\left(\mathbf{r}_{0}, \varepsilon_{F}+e V\right) \approx \Gamma_{0}\left(\varepsilon_{F}\right)+\frac{\partial \Gamma_{0}}{\partial \varepsilon_{F}} e V, \quad \frac{\partial \Gamma_{0}}{\partial \varepsilon_{F}} \sim \frac{1}{\varepsilon_{F}} \frac{r_{0}}{\lambda_{F}}
$$

and when the product $\left(e V / \varepsilon_{F}\right)\left(r_{0} / \lambda_{F}\right) \gg 1$ clearly the conductance (40) is an oscillatory function of the voltage $V$. Note that if the inequality $e V \ll \varepsilon_{F}$ is not satisfied, the value of the conductance (41) as well as the amplitude of its oscillations depend on $V$. The periods of oscillations are defined by the energy dependence of the function $\Gamma_{0}\left(\varepsilon, \mathbf{r}_{0}\right)$ and they remain the same for any voltage. Below presenting formulas for the conductance in different cases we do not expand arguments of oscillatory functions in the parameter $e V / \varepsilon_{F}$. The obtained results properly describe the total conductance at $e V \ll \varepsilon_{F}$ and also can be used for the analysis of periods of oscillations at $e V \leqslant \varepsilon_{F}$. In Eq. (40) the $\Lambda_{s}^{\text {as }}\left(\mathbf{r}_{0}, \varepsilon_{F}, e V\right)$ denotes the function (32), in which $\Gamma_{0}=\Gamma_{0}\left(\mathbf{r}_{0}, \varepsilon_{F}+e V\right)$. Equation (40) shows that for a tunnel junction of small size the amplitude and period of conductance oscillations depend on the local geometry FS in those points for which the velocity is directed along the vector $\mathbf{r}_{0}$.

In the case of a convex FS there is only one stationary phase point satisfying Eq. (31) which allows simplifying the expression for the conductance (40). The oscillating part of the conductance, $\Delta G$, can be written as

$$
\begin{aligned}
\frac{\Delta G}{G_{0}}= & \frac{g}{2\left\langle\left(v_{z}^{(+)}\right)^{2}\right\rangle_{\varepsilon_{F}} \nu\left(\varepsilon_{F}\right)(2 \pi \hbar)^{3} r_{0}^{2}}\left(\frac{\left[v_{z}^{(+)}\left(\varepsilon, \mathbf{p}_{t}\right)\right]^{2}}{\sum_{i, k=x, y, z} A_{i k} n_{0 i} n_{0 k}}\right. \\
& \left.\times \sin \left[2 \Gamma_{0}\left(\mathbf{r}_{0}, \varepsilon_{F}+e V\right)\right]\right)_{\mathbf{p}_{t}=\mathbf{p}_{t}^{(\mathrm{st})}},
\end{aligned}
$$

where $\mathbf{n}_{0}=\mathbf{r}_{0} / r_{0}$. The phase of the oscillations in the conductance, $2 \Gamma_{0}$, is determined by the phase that the electron accumulates along the trajectory from the contact to the defect and back 


$$
2 \Gamma_{0}\left(\mathbf{r}_{0}, \varepsilon\right)=\frac{2}{\hbar} \mathbf{p}^{(\mathrm{st})} \mathbf{r}_{0}
$$

where $2\left|\mathbf{p}^{(\mathrm{st})}\right|$ is the chord connecting the two points on the surface of constant energy for which the velocities are antiparallel and aligned with the vector $\mathbf{r}_{0}$.

If the direction from the contact to the defect coincides with a direction of electron velocities for a flat part of the FS, we should use asymptotic expression (39) for the function $\Lambda_{\mathrm{fl}}^{\mathrm{as}}\left(\mathbf{r}_{0}, \varepsilon\right)$ when calculating the conductance (40). For this case the oscillating part of the conductance, $\Delta G$, is given by

$$
\frac{\Delta G}{G_{0}}=\frac{\pi g S_{\mathrm{fl}}^{2} v_{z 0}^{2}}{\left\langle\left(v_{z}^{(+)}\right)^{2}\right\rangle_{\varepsilon_{F}}\left|\mathbf{v}_{0}\right|^{2} \nu\left(\varepsilon_{F}\right)(2 \pi \hbar)^{4}} \sin \left(\frac{2\left(\varepsilon_{F}+e V\right) r_{0}}{\hbar\left|\mathbf{v}_{0}\right|}\right) .
$$

Note that $r_{0} /\left|\mathbf{v}_{0}\right|=z_{0} / v_{z 0}$. In the case when the FS has a flat part, the amplitude of the conductance oscillations for those special directions does not depend on the distance between the contact and the defect.

In the next sections we shall consider two models of anisotropic FSs. The first model, of an ellipsoidal FS, illustrates the main features of the conductance oscillations for metals with a convex FS, i.e., with positive Gaussian curvature. The FS of the second model has the shape of a corrugated cylinder, which illustrates the effects of sign inversion of the curvature and the presence of open directions of the FS. These two models allow us to obtain dependencies of the conductance on the applied voltage and on the defect position in analytical form.

\section{ELLIPSOIDAL FERMI SURFACE}

For an ellipsoidal FS the Schrödinger Eq. (2) can, in fact, be solved exactly in the limit $R \rightarrow 0$ and the wave function (19) and the conductance (25) can be found for arbitrary distances between the contact and the defect. For this FS the dependence of the electron energy $\varepsilon$ on the momentum $\mathbf{p}$ is given by relation

$$
\varepsilon(\mathbf{p})=\frac{1}{2} \sum_{i, k=x, y, z} \frac{p_{k} p_{i}}{m_{i k}}
$$

where $p_{i}$ are the components of the electron momentum $\mathbf{p}$, $1 / m_{i k}$ are constants representing the components of the inverse effective mass tensor $\mathbf{m}^{-1}$. The tensor $\mathbf{m}^{-1}$ can be diagonalized to the form $\left\{\mathbf{m}^{-1}\right\}_{i k}=m_{i}^{-1} \delta_{i k}$ so that the momentum-space lengths $\sqrt{2 \varepsilon_{F} m_{i}}$ correspond to the semiaxes of the FS ellipsoid $\varepsilon(\mathbf{p})=\varepsilon_{F}$.

For the ellipsoidal FS in absence of a defect (zeroth approximation) the wave function $\varphi^{(+)}(\mathbf{r})(27)$ can be obtained by integration of Eq. (27) over momentum,

$$
\varphi^{(+)}(\mathbf{r})=t\left(\varepsilon, \mathbf{p}_{t}\right) \frac{\sqrt{2}(\varepsilon)^{3 / 2} z R^{2}}{\hbar^{3} \sqrt{\operatorname{det} \mathbf{m}^{-1}}} \frac{e^{i \Gamma_{0}\left[1-i \Gamma_{0}(\mathbf{r})\right]}}{\Gamma_{0}^{3}(\mathbf{r})},
$$

where

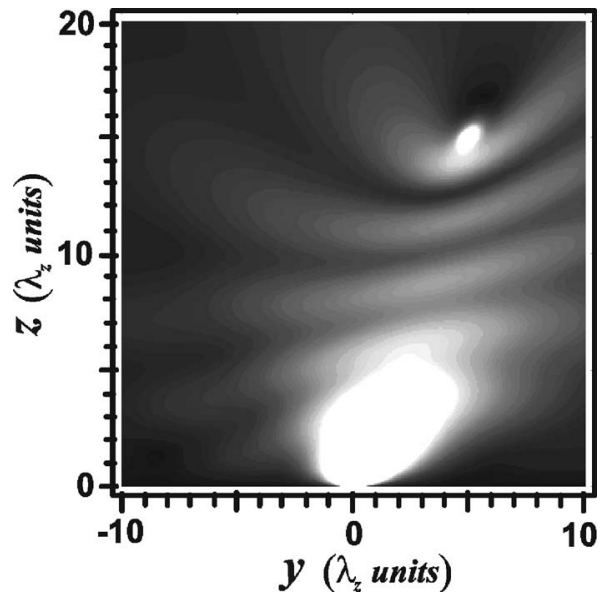

FIG. 1. Gray-scale plot of the modulus of the wave function in the plane $x=0$ for an ellipsoidal FS. The shape of the FS is defined by the mass ratios $m_{x} / m_{z}=1, m_{y} / m_{z}=3$, and the long axis of the ellipsoid is rotated by $\pi / 4$ around the $x$ axis, away from the $y$ axis. The coordinates are measured in units of $\lambda_{z}=\hbar / \sqrt{2 m_{z z} \varepsilon}$. The position of the defect is $\mathbf{r}_{0}=(0,5,15)$. An interference pattern is visible of partial waves reflected from the impurity with those emanating from the contact that is influenced by the anisotropy of the electronic band structure.

$$
\Gamma_{0}(\varepsilon, \mathbf{r})=\frac{r}{\hbar} \sqrt{2 \varepsilon \sum_{i, k=x, y, z} \mu_{i k} n_{i} n_{k}}
$$

and

$$
t\left(\varepsilon, \mathbf{p}_{t}\right)=\frac{\hbar}{i U m_{z z}} \sqrt{2 m_{z z} \varepsilon-m_{z z} \sum_{i, k=x, y} \frac{p_{i} p_{k}}{m_{i k}}+\left(m_{z z} \sum \frac{p_{i}}{m_{z i}}\right)^{2}} .
$$

Here, $\mu_{i k}$ are the elements of the mass tensor $\boldsymbol{\mu}$, which is the inversive to the tensor $\mathbf{m}^{-1}\left(\boldsymbol{\mu \mathbf { m } ^ { - 1 }}=\mathbf{I}\right.$, with $\mathbf{I}$ the unitary tensor; $\left.\mu_{i k}=A_{i k} / \operatorname{det} \mathbf{m}^{-1}\right)$. The Green function (18) takes the form

$$
\begin{aligned}
G_{0}^{+}\left(\mathbf{r}, \mathbf{r}^{\prime} ; \varepsilon\right)= & \frac{2 \pi \sqrt{2 \varepsilon}}{\hbar^{3} \sqrt{\operatorname{det} \mathbf{m}^{-1}}}\left(\frac{\exp \left(i \sqrt{\Gamma_{0}^{2}\left(\mathbf{r}-\mathbf{r}^{\prime}\right)+\frac{8 \varepsilon m_{z z} z z^{\prime}}{\hbar^{2}}}\right)}{\sqrt{\Gamma_{0}^{2}\left(\mathbf{r}-\mathbf{r}^{\prime}\right)+\frac{8 \varepsilon m_{z z} z z^{\prime}}{\hbar^{2}}}}\right. \\
& \left.-\frac{\exp \left[i \Gamma_{0}\left(\mathbf{r}-\mathbf{r}^{\prime}\right)\right]}{\Gamma_{0}\left(\mathbf{r}-\mathbf{r}^{\prime}\right)}\right) .
\end{aligned}
$$

Using Eqs. (47) and (50) we obtain the wave function (19) to first approximation in the strength of the impurity potential. The modulus of this wave function is illustrated in Fig. 1 for a plane normal to the interface passing through the impurity and the contact.

The conductance in the limit of low temperatures, $T \rightarrow 0$, is obtained from Eqs. (23) and (25) by integration over all directions of the momentum $\mathbf{p}$ and integration over the space coordinate $\boldsymbol{\rho}$ in a plane $z=$ const $\left(z>z_{0}\right)$, retaining only terms 


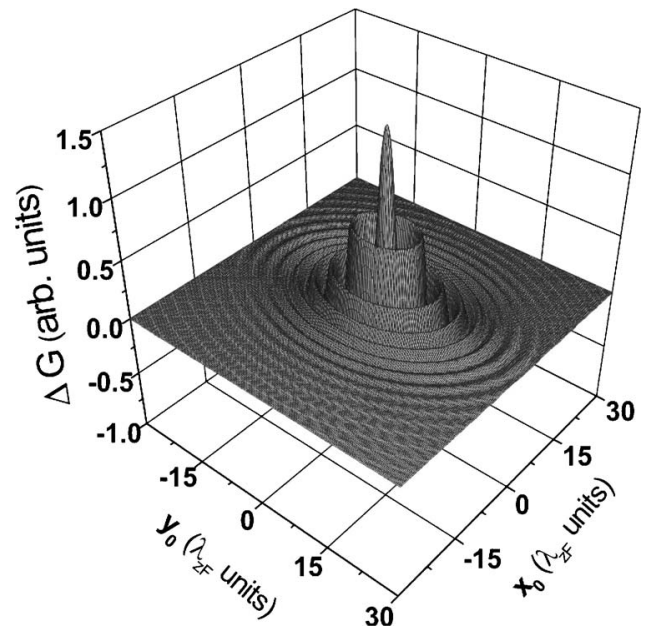

FIG. 2. Dependence of the oscillatory part of the conductance, $\Delta G$, as a function of the position of the defect $\boldsymbol{\rho}_{0}$ in the plane $z$ $=z_{0}$ for the same shape and orientation of the ellipsoidal FS as in Fig. 1. The coordinates are measured in units $\lambda_{z F}=\hbar / \sqrt{2 m_{z z} \varepsilon_{F}}$ and the defect sits at $z_{0}=5$. The figure shows that $\Delta G$ is an oscillatory function of the defect position that reflects the ellipsoidal form of the FS and the oscillations are largest when the defect is placed in the position $\boldsymbol{\rho}_{00}$, defined by Eq. (52).

to first order in $g$ (i.e., ignoring multiple scattering at the impurity site),

$$
\begin{aligned}
G^{\mathrm{ell}}(V)= & G_{0}^{\mathrm{ell}}\left\{1-\frac{12 \pi^{2} g z_{0}^{2}\left(2 \varepsilon_{F}\right)^{3 / 2}}{\hbar^{5} \sqrt{m_{z z}} \operatorname{det}\left(\mathbf{m}^{-1}\right)} \frac{1}{\Gamma_{0}^{4}\left(\varepsilon_{F}, \mathbf{r}_{0}\right)}\right. \\
& \times\left[\frac{1}{2}\left(1-\frac{1}{\Gamma_{0}^{2}\left(\varepsilon_{F}, \mathbf{r}_{0}\right)}\right) \sin 2 \Gamma_{0}\left(\varepsilon_{F}+e V, \mathbf{r}_{0}\right)\right. \\
& \left.\left.+\frac{1}{\Gamma_{0}\left(\varepsilon_{F}, \mathbf{r}_{0}\right)} \cos 2 \Gamma_{0}\left(\varepsilon_{F}+e V, \mathbf{r}_{0}\right)\right]\right\},
\end{aligned}
$$

with $\Gamma_{0}(\varepsilon, \mathbf{r})$ given by $(44)$.

The amplitude of the conductance oscillations is maximal when $\Gamma_{0}$ is minimal. For a fixed depth $z_{0}$ this minimum occurs when the defect position $\boldsymbol{\rho}_{0}$ is in the point $\boldsymbol{\rho}_{00}$ with respect to the point contact at $\mathbf{r}=0$, where

$$
\boldsymbol{\rho}_{00}=z_{0}\left(\begin{array}{l}
m_{z z} / m_{z x} \\
m_{z z} / m_{z y}
\end{array}\right) \text {. }
$$

The minimal value of the phase then becomes

$$
\Gamma_{00}=\Gamma_{0}\left(\varepsilon_{F}+e V, \boldsymbol{\rho}_{00}, z_{0}\right)=\frac{1}{\hbar} z_{0} \sqrt{2\left(\varepsilon_{F}+e V\right) m_{z z}} .
$$

The phase $\Gamma_{00}$ corresponds to the extremal value of the chord of the FS in the direction normal to the interface. $G_{0}^{\text {ell }}$ in Eq. (51) is the conductance in the absence of a defect $(g=0)$,

$$
G_{0}^{\text {ell }}=\frac{2 e^{2} R^{4} \varepsilon_{F}^{3}}{9 \pi \hbar^{3} U^{2} \sqrt{\operatorname{det}\left(\mathbf{m}^{-1}\right)} \sqrt{m_{z z}}} .
$$

Figure 2 shows a plot of the oscillatory part of the con-

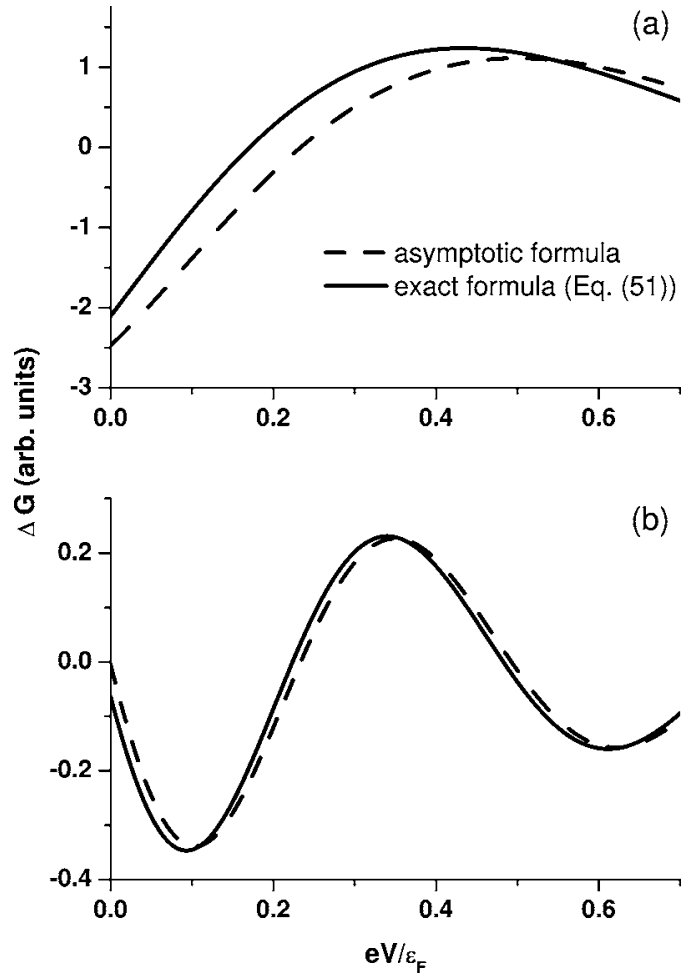

FIG. 3. Comparison of the oscillating part of the conductance for an ellipsoidal FS calculated in the point $\boldsymbol{\rho}_{0}$ (52) of the maximum amplitude by using the asymptotic (dashed curve) and exact (solid curve) formulas. The depths of the defect are $z_{0}=4$ (a) and $z_{0}=10$ (b) in units of $\lambda_{z F}$.

ductance, $\Delta G=G^{\text {ell }}(0)-G_{0}^{\text {ell }}$, Eq. (51), for the contact as a function of the position of the defect, $\boldsymbol{\rho}_{0}$, in the limit of low voltage, $V \rightarrow 0$.

For the ellipsoidal model FS the wave function and conductance have been obtained exactly, within the framework of the model. For large distances $r$ and $r_{0}$ they transform into the asymptotic expressions, Eqs. (37) and (40). We do not present the asymptotic form explicitly but it agrees to within a term proportional to $\Gamma_{0}^{-4}$ to the exact from, Eq. (51). In Fig. 3 we compare the results for the calculations of the conductance by using the exact (51) and asymptotic (40) expressions. The figure confirms that for relatively small distances [Fig. 3(a)] the asymptotic formula still qualitatively describes the conductance very well and that for larger distances [Fig. 3(b)] the two results are in a good agreement. The parameters for the FS in Figs. 2 and 3 are the same as those for Fig. 1.

\section{OPEN FERMI SURFACE}

The second model FS we want to discuss has the form of a corrugated cylinder (Fig. 4), which is open along the direction $p_{\|}$,

$$
\varepsilon(\mathbf{p})=\frac{p_{\perp}^{2}}{2 m}+\varepsilon_{1} \sin ^{2} \frac{p_{\|} b}{2}, \quad-\frac{\pi}{b} \leqslant p_{\|} \leqslant \frac{\pi}{b}, \quad \varepsilon>\varepsilon_{1},
$$

where $2 \pi / b$ is the size of the Brillouin zone, and $m$ is an effective mass. We further impose that the momentum per- 


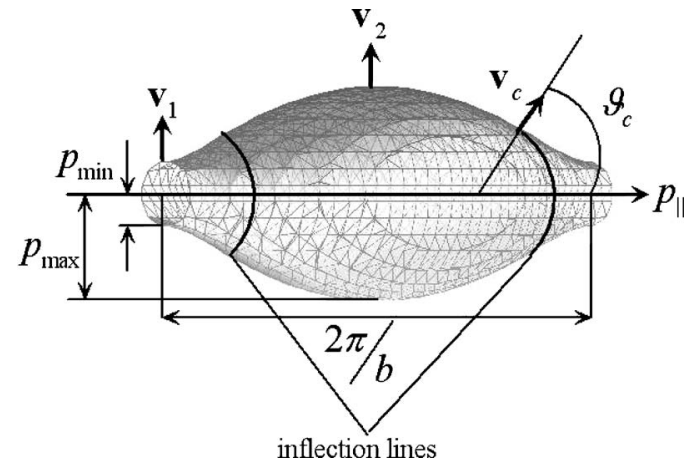

FIG. 4. Model of an open FS. The figure illustrates an existence of two points on the FS, at which velocities are parallel. The inflection lines corresponding to the zero Gaussian curvature are shown.

pendicular to the symmetry axis of the FS remains finite,

$$
p_{\perp \max } \leqslant p_{\perp} \leqslant p_{\perp \min }
$$

where

$$
p_{\perp \max }(\varepsilon)=\sqrt{2 m \varepsilon}, \quad p_{\perp \min }(\varepsilon)=\sqrt{2 m\left(\varepsilon-\varepsilon_{1}\right)}
$$

are the maximal and minimal radii of the cylindrical surface, respectively. As a consequence of rotational symmetry the Gaussian curvature $K$ (34) of the surface depends only on $p_{\perp}$. The central part of the surface (belly) has a positive curvature $K>0$ while the ends near the Brillouin zone boundary (necks) have negative curvature. In the direction perpendicular to the symmetry axis there are two partial waves propagating with different parallel velocities, $\mathbf{v}_{1}$ and $\mathbf{v}_{2}$, belonging to the parts of FS having opposite sign of $K$. Rotating away from the perpendicular direction towards the axis the two solutions persist but the two corresponding points on the FS move closer together until they merge at the curve defined by $K=0$, the inflection line. For directions beyond this angle (i.e., for $\theta<\theta_{c}$ in Fig. 4) no propagating wave solutions exist. On the inflection line a unique solution with velocity $\mathbf{v}_{c}$ is found. For $\theta>\theta_{c}$ there are two stationary phase points $p_{\perp s}(s=1,2)$ that satisfy Eq. (31), corresponding with two different velocities $\mathbf{v}^{(+)}\left(p_{\perp s}\right)$ directed along the radius vector $\mathbf{r}$. The larger value, $p_{\perp 2}$, belongs to the belly of the FS $(K>0)$ and the smaller one, $p_{\perp 1}$, belongs to the neck $(K<0)$. At the inflection line of the surface we have

$$
\left.\frac{\partial^{2} p_{\|}\left(\varepsilon, p_{\perp}\right)}{\partial p_{\perp}^{2}}\right|_{p_{\perp}=p_{\perp 0}}=0,
$$

which defines the value of perpendicular momentum $p_{\perp 0}$. From this condition we obtain

$$
p_{\perp 0}=\sqrt{p_{\perp \max } p_{\perp \min }} .
$$

The cone inside of which no propagating states exist is defined by the condition

$$
\left.\frac{v_{\|}}{v_{\perp}}\right|_{p_{\perp}=p_{\perp 0}}=\cot \vartheta_{c}=\frac{b}{2}\left(p_{\perp \max }-p_{\perp \min }\right) \operatorname{sgn}\left(v_{\|}\right),
$$

where the components of the velocity $v_{\|}$and $v_{\perp}$ are given by

$$
v_{\|}=\frac{\varepsilon_{1} b}{2} \sin \left(p_{\|} b\right), \quad v_{\perp}=\frac{p_{\perp}}{m} .
$$

In spite of the simplicity of the model FS (55), the integrals in Eqs. (19) and (25), cannot be evaluated analytically. We can only discuss the asymptotic behavior for $r_{0} \gg \lambda_{F}$. Qualitatively this result should also be valid for $r_{0}>\lambda_{F}$. For the directions that have two stationary phase points, having opposite signs of the Gaussian curvature, Eq. (40) acquires the form

$$
\begin{aligned}
G^{\mathrm{op}}= & G_{0}^{\mathrm{op}}\left[1+\frac{g \cos ^{2} \vartheta\left(\mathbf{n}_{0}\right)}{(2 \pi \hbar)^{3} \hbar\left\langle\left(v_{z}^{(+)}\right)^{2}\right\rangle \nu\left(\varepsilon_{F}\right) r_{0}^{2}}\right. \\
& \times \sum_{s, s^{\prime}=1,2} \frac{1}{\sqrt{\left|K_{0}^{(s)}\left(\varepsilon_{F}, \mathbf{n}_{0}\right) K_{0}^{\left(s^{\prime}\right)}\left(\varepsilon_{F}, \mathbf{n}_{0}\right)\right|}} \\
& \times \cos \left(\Gamma_{0}^{(s)}\left(\varepsilon_{F}+e V, \mathbf{r}_{0}\right)\right. \\
& \left.+\frac{\pi}{2}(1-s)\right) \sin \left(\Gamma_{0}^{\left(s^{\prime}\right)}\left(\varepsilon_{F}+e V, \mathbf{r}_{0}\right)\right. \\
& \left.\left.+\frac{\pi}{2}\left(1-s^{\prime}\right)\right)\right]
\end{aligned}
$$

where $G_{0}^{\mathrm{op}}$ is the conductance of the contact without defect given by Eq. (41), $\Gamma_{0}^{(s)}(\varepsilon, \mathbf{r})=\Gamma\left(\mathbf{p}_{t, s}^{(\mathrm{st})}, \mathbf{r}\right)$, and $K_{0}^{(s)}(\varepsilon, \mathbf{n})$ $=K\left(\mathbf{p}_{t, s}^{(\mathrm{st})}, \varepsilon\right)$.

The appearance of the conductance oscillations depends strongly on the orientation of the FS with respect to the interface. Below we will consider two specific orientations, having the axis of the FS either perpendicular or parallel to the interface.

\section{A. Direction of open FS perpendicular to the interface}

When the isoenergy surface is open along the contact axis $z$ the components of the momenta in Eq. (55) are $p_{\perp}$ $=\sqrt{p_{x}^{2}+p_{y}^{2}}$ and $p_{\|}=p_{z}$. In this case the conductance of the clean contact (without defect) becomes

$$
G_{0}^{\mathrm{op}}=\frac{\pi e^{2} R^{4} m^{2} \varepsilon_{1}^{4} b^{2}}{8 \hbar^{3} U^{2}}
$$

From Eq. (31) the stationary phase points for the iso-energy surface are

$$
\begin{aligned}
p_{\perp s}^{2}= & \frac{1}{2}\left[p_{\perp \text { max }}^{2}+p_{\perp \text { min }}^{2}-\frac{4}{b^{2}} \cot ^{2} \vartheta+(\right. \\
& \left.-1)^{s} \sqrt{\left(p_{\perp \text { max }}^{2}+p_{\perp \text { min }}^{2}-\frac{4}{b^{2}} \cot ^{2} \vartheta\right)^{2}-4 p_{\perp \text { max }}^{2} p_{\perp \text { min }}^{2}}\right] .
\end{aligned}
$$

The angle $\vartheta=\arccos (z / r)$ is defined by the direction of the radius vector $\mathbf{r}$. The Gaussian curvature $K_{0}$ and the phase $\Gamma_{0}$ in the points (64) are given by the relations 


$$
\begin{gathered}
K_{0}^{(s)}(\varepsilon, \mathbf{n})=\frac{b^{2}(-1)^{s} \sin ^{4} \vartheta}{4 p_{\perp s}^{2}} \sqrt{\left(p_{\perp \max }^{2}+p_{\perp \min }^{2}-\frac{4}{b^{2}} \cot ^{2} \vartheta\right)^{2}-4 p_{\perp \max }^{2} p_{\perp \min }^{2}} \\
\Gamma_{0}^{(s)}(\varepsilon, \mathbf{r})=\frac{1}{\hbar} p_{\perp s} \sqrt{x^{2}+y^{2}}+\frac{2 z}{\hbar b} \arcsin \sqrt{\frac{2 m \varepsilon-p_{\perp s}^{2}}{2 m \varepsilon_{1}}} . \quad G_{\max }^{\mathrm{op}}=G_{0}^{\mathrm{op}}\left[1-\frac{C g}{m \varepsilon_{1}}\left(\frac{b^{2}}{\hbar^{4} z_{0}^{5}}\right)^{1 / 3} \sqrt{p_{\perp \max } p_{\perp \min }\left(p_{\perp \max }\right.}\right. \\
\left.\left.-p_{\perp \min }\right)^{3} \sin \left(2 \Gamma_{00}-\frac{5 \pi}{6}\right)\right],
\end{gathered}
$$

The angle $\vartheta$ in Eqs. (64) and (65) is contained within the interval $\vartheta_{c} \leqslant \vartheta \leqslant \pi / 2$, where the $\vartheta_{c}$ is given by Eq. (60).

The modulus of the wave function is plotted in Fig. 5. For the calculation of the wave function, Eq. (37), we used formulas (65) for the curvature and (66) for the phase in the asymptotic expression for the integral $\Lambda^{\text {as }}$ (32). Although, strictly speaking Eq. (37) is not applicable in the vicinity of the contact and near the defect, nor inside the classically inaccessible region, Fig. 5 illustrates the main features of this problem. One observes the interference of the two partial waves with different velocities, the existence of a forbidden cone, the anisotropy of the waves scattered by the defect, and the enhanced wave function amplitude near the edge of the forbidden cone.

At the inflection lines, where $K=0$ and $\theta$ is given by Eq. (60), the square root in Eq. (64) is equal to zero. For this direction two stationary phase points merge and the electron velocity is directed along the cone of the classically forbidden region. The asymptotic expression for the conductance (62) diverges at these points, which implies that the third derivative of the phase, Eq. (29), with respect to $p_{\perp}$ must be taken into account. When the vector $\mathbf{r}_{0}$ connecting the point contact to the defect lies along the cone of the forbidden region the conductance oscillations have maximal amplitude and the conductance takes the form

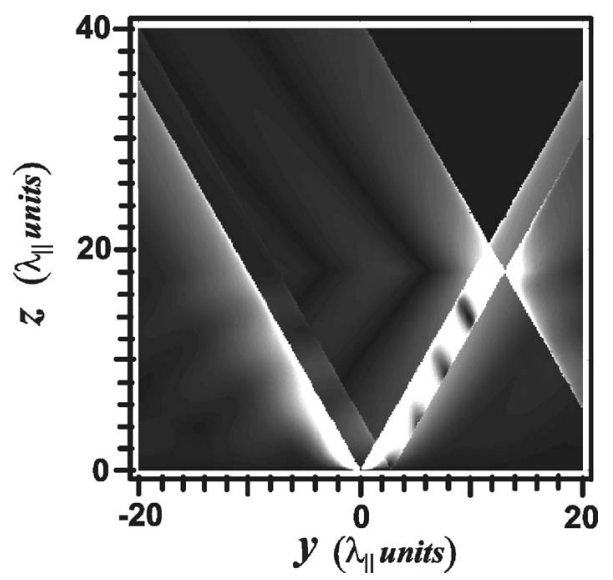

FIG. 5. Gray-scale plot of the modulus of the wave function in the plane $x=0$ for a warped cylindrical FS having the open direction along the contact axis $z$. The coordinates are measured in units of $\lambda_{\|}=\hbar / \sqrt{2 m \varepsilon}$. The parameters used in the model are $\varepsilon_{1} / \varepsilon=0.9$, $b \sqrt{2 m \varepsilon}=5.2$, and a defect sits at $\mathbf{r}_{0}=(0,13,18)$. where

$$
\begin{aligned}
\Gamma_{00}= & \Gamma_{0}\left(p_{\perp 0}, z_{0}\right)=\frac{2 z_{0}}{b \hbar}\left(\frac{\sqrt{p_{\perp \max } p_{\perp \mathrm{min}}}}{p_{\perp \max }-p_{\perp \mathrm{min}}}\right. \\
& +\left.\arcsin \sqrt{\left.\frac{p_{\perp \max }^{2}-p_{\perp \max } p_{\perp \min }}{2 m \varepsilon_{1}}\right)}\right|_{\varepsilon=\varepsilon_{F}+e V},
\end{aligned}
$$

and $C$ is a numerical constant, $C \simeq 1.97 \times 10^{-4}$. The energy dependencies of $p_{\perp \max }$ and $p_{\perp \text { min }}$ are given by Eq. (57).

Figure 6 shows a plot of the oscillatory part $\Delta G=G^{\mathrm{op}}(0)$ $-G_{0}^{\mathrm{op}}$ of the conductance $G^{\mathrm{op}}(0)$, Eq. (62), as a function of the lateral position of the defect $\boldsymbol{\rho}_{0}$ for a fixed distance $z_{0}$ from the interface. The oscillation pattern has a dead region in the center, corresponding to defect positions inside the classically inaccessible part of the metal for electrons injected by the point contact. This region is defined by the cone (60) and its radius $\rho_{00}=z_{0} / \cot \vartheta_{c}$ depends on the depth of the defect under surface. The oscillations in the conductance are largest when the defect is placed at the edge of the cone $\rho_{0}$

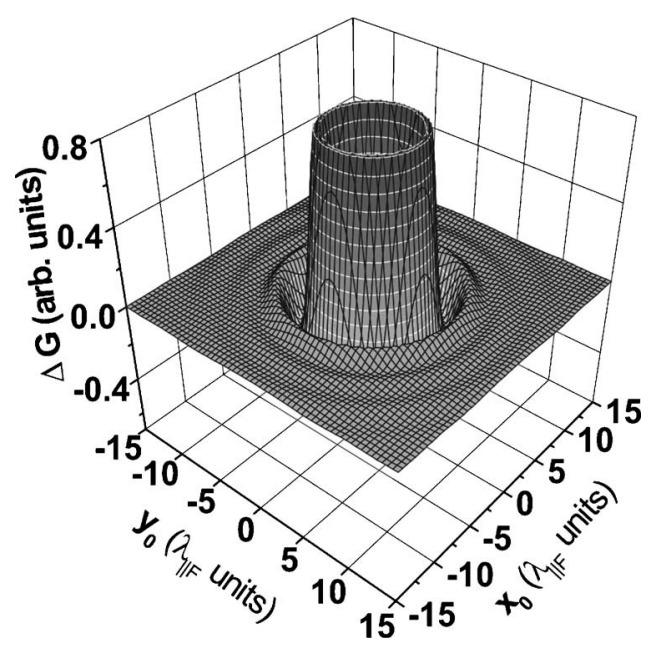

FIG. 6. Dependence of the oscillatory part $\Delta G$ of the conductance, as a function of the lateral position of the defect $\boldsymbol{\rho}_{0}$ in the plane $z=z_{0}$. The open direction of the FS is oriented perpendicular to the interface. The coordinates are measured in units of $\lambda_{\| F}$ $=\hbar / \sqrt{2 m \varepsilon_{F}}$. The parameters used for the model FS (55) are $\varepsilon_{1} / \varepsilon_{F}$ $=0.9, b \sqrt{2 m \varepsilon_{F}}=6$, and a defect sits at a depth of $z_{0}=11$. In a central part of the plotted dependence $\Delta G\left(x_{0}, y_{0}\right)$ the "dead region," in which $\Delta G=0$, is visible. 
$=\rho_{00}$. In this case the defect is positioned in a direction of velocity belonging to the inflection line of the FS and the electron flux in this direction is maximal.

\section{B. Direction of open FS parallel to the interface}

The second orientation we want to discuss is that with the FS (55) having its open direction (the axis) parallel to interface, with $\mathbf{p}_{\perp}=\left(p_{x}, p_{z}\right)$ and $p_{\|}=p_{y}$. The existence of a classically inaccessible region for this geometry leads to a strongly anisotropic current density in the $x y$ plane. The expression for the point contact conductance without defect $G_{0}^{\text {op }}$ becomes

$$
G_{0}^{\mathrm{op}}=\frac{e^{2} R^{4} \pi\left(2 \varepsilon-\varepsilon_{1}\right)^{2}}{4 \hbar^{3} b^{2} U^{2}} .
$$

The expressions for the phase, Eq. (44), and the Gaussian curvature, Eq. (34), now read,

$$
\left.\Gamma_{0}^{(s)}(\varepsilon, \mathbf{r})=\frac{1}{\hbar}\left(\sqrt{\left(x^{2}+z^{2}\right)\left[2 m\left(\varepsilon-\varepsilon_{1} \lambda_{s}\right)\right.}\right]+\frac{2|y|}{b} \arcsin \sqrt{\lambda_{s}}\right),
$$

and

$$
K_{0}^{(s)}(\varepsilon, \mathbf{n})=(-1)^{s} \frac{\varepsilon_{1} b^{2} \sin ^{4} \theta}{4\left(\varepsilon-\varepsilon_{1} \lambda_{s}\right)} \sqrt{\left(1+\frac{2 \cot ^{2} \theta}{m \varepsilon_{1} b^{2}}\right)^{2}-\frac{8 \varepsilon \cot ^{2} \theta}{m \varepsilon_{1}^{2} b^{2}}},
$$

respectively. For this geometry we use spherical coordinates, with $\theta$ the angle between the vector $\mathbf{r}$ and the $y$ axis. The variables $\lambda_{1,2}$ have been obtained from Eq. (31),

$\lambda_{s}=\frac{1}{2}\left[1+\frac{2 \cot ^{2} \theta}{m \varepsilon_{1} b^{2}}+(-1)^{s} \sqrt{\left(1+\frac{2 \cot ^{2} \theta}{m \varepsilon_{1} b^{2}}\right)^{2}-\frac{8 \varepsilon \cot ^{2} \theta}{m \varepsilon_{1}^{2} b^{2}}}\right]$.

The first stationary phase point, $\lambda_{1}$, corresponds to a positive Gaussian curvature and the second one, $\lambda_{2}$, to a negative curvature. For $\theta=\vartheta_{c}$, Eq. (60), they become equal,

$$
\lambda_{1}=\lambda_{2}=\frac{1}{2 m \varepsilon_{1}}\left(p_{\perp \text { max }}^{2}-\sqrt{p_{\perp \text { max }}^{2} p_{\perp \text { min }}^{2}}\right),
$$

and the curvature (70) vanishes.

Figure 7, acquired by using Eqs. (37), (69), and (70), illustrates the interference of waves with different velocities the electrons emerging from the contact and the interference with the waves scattered by the defect.

Figure 8 shows a plot of the oscillatory part $\Delta G$ of the conductance $G^{\mathrm{op}}(0)$ as a function of the lateral position of the defect $\boldsymbol{\rho}_{0}$ for a fixed distance $z_{0}$ from the interface. In this case two dead regions appear symmetrically with respect to the center of the oscillation pattern along the open direction of the FS. The center of the pattern corresponds to a defect sitting on the axis of the contact, $\boldsymbol{\rho}_{0}=0$, for which $\lambda_{1}=0$, $\lambda_{2}=1, \sin \theta=1$, and $\cos \varphi=1$. At this point Eq. (40) takes the form

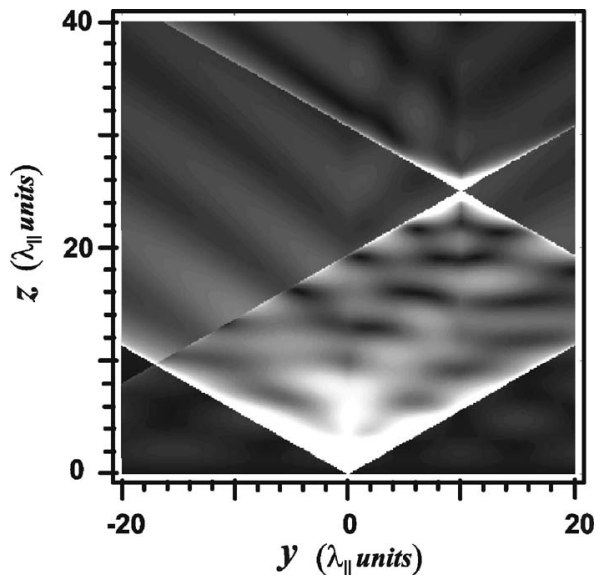

FIG. 7. Gray-scale plot of the modulus of the wave function in the plane $x=0$ for the warped cylindrical Fermi surface with the open direction along the $y$ axis and parallel to the plane of interface. The coordinates are measured in units of $\lambda_{\|}$. The Fermi surface parameters are $\varepsilon_{1} / \varepsilon=0.9, b \sqrt{2 m \varepsilon}=5.2$, and the defect position is $\mathbf{r}_{0}=(0,10,25)$. For this geometry the classically inaccessible region is found near the interface to both sides of the contact.

$$
\begin{aligned}
G^{\mathrm{op}}(V)= & G_{0}^{\mathrm{op}}\left\{1+\frac{4 g}{z_{0}^{2} \pi^{2} \hbar\left(p_{\perp \text { max }}^{2}+p_{\perp \text { min }}^{2}\right) \varepsilon_{1} b}\right. \\
& \times\left[p_{\perp \min }^{2} \sin \left(\frac{2 p_{\perp \min }\left(\varepsilon_{F}+e V\right) z_{0}}{\hbar}\right)\right. \\
& -p_{\perp \max }^{2} \sin \left(\frac{2 p_{\perp \max }\left(\varepsilon_{F}+e V\right) z_{0}}{\hbar}\right) \\
& +2 p_{\perp \max } p_{\perp \min } \\
& \left.\left.\times \cos \left(\frac{p_{\perp \max }\left(\varepsilon_{F}+e V\right) z_{0}+p_{\perp \min }\left(\varepsilon_{F}+e V\right) z_{0}}{\hbar}\right)\right]\right\} .
\end{aligned}
$$

\section{DISCUSSION}

We have analyzed the oscillatory voltage dependence of the conductance of a tunnel junction in the presence of an elastic scattering center located inside the bulk for metals with an anisotropic FS. These oscillations result from electron waves being scattered by the defect and reflected back by the contact, interfering with electrons that are directly transmitted through the contact. The introduction of anisotropic electron movement beams the following implication: several points on the FS may share the same direction of the group velocity vector $\mathbf{v}$ whereas other directions for $\mathbf{v}$ can be absent. Two nonspherical shapes for the FS have been investigated: the ellipsoid and the corrugated cylinder (open surface).

Contrary to the case of a spherical FS (Ref. 8) in the ellipsoidal model (46) the center of the conductance oscillation pattern does not need to coincide with the actual position of the defect but is displaced over a vector $\rho_{00}$ $=z_{0}\left(m_{z z} / m_{z x}, m_{z z} / m_{z y}\right)$. When the STM tip is placed at this point the oscillatory part of the conductance is given by 


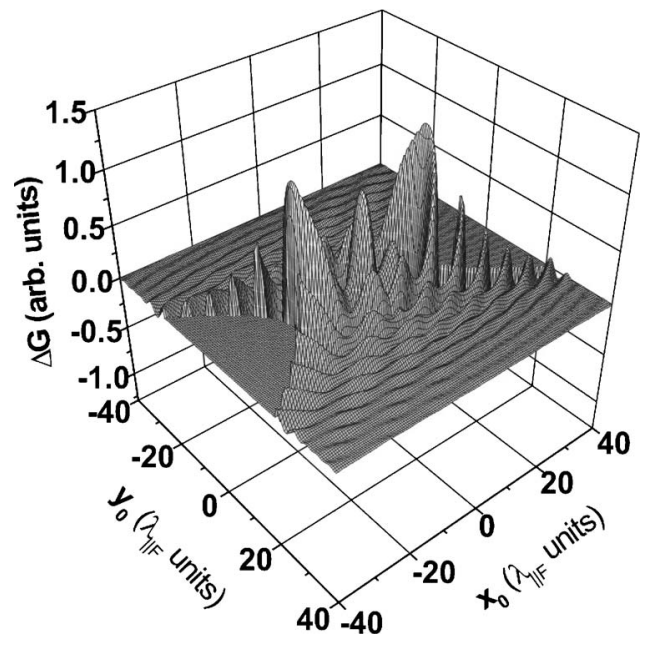

FIG. 8. Dependence of the oscillatory part $\Delta G$ of the conductance, as a function of the lateral position of the defect $\boldsymbol{\rho}_{0}$ in the plane $z=z_{0}$. The open direction of the FS is oriented parallel to the interface along the $y$ direction. The coordinates are measured in units of $\lambda_{\| F}$. The parameters used for the model FS (55) are $\varepsilon_{1} / \varepsilon_{F}=0.9, b \sqrt{2 m \varepsilon_{F}}=6$, and a defect sits at a depth of $z_{0}=11$.

$$
\Delta G(V) \propto \sin \left(\frac{2}{\hbar} z_{0} \sqrt{2\left(\varepsilon_{F}+e V\right) m_{z z}}\right) .
$$

The oscillation period depends on $1 / m_{z z}$, the component of the tensor of inversive mass (35) for motion in the $z$ direction, and on the depth $z_{0}$ of the defect. This allows us in principle to map out the positions of defects, as long as the shape of the FS is known. Apart from the period of the oscillations, there is also information in the amplitude. Since short periods should correspond to small amplitudes this may be used for a test of consistency. However, quantitatively the amplitude is also influenced by unknown factors such as the defect scattering efficiency. The ellipsoidal FS is exceptional in that the problem can be solved exactly. This allows us to compare the calculation with the asymptotic approximation, and this shows that the approximation works very well for distances larger than $\lambda_{F}$.

In the case of the corrugated cylinder (55) the open necks cause cones with opening angle $2 \vartheta_{c}$ (defined by the inflection line of the FS) to be classically inaccessible. If the orientation is such that the open direction is orthogonal to the surface this will result in a dead region with radius $z_{0} / \cot \vartheta_{c}$ (60) where no conductance fluctuations can be observed. Thus, by measuring the size of this dead region we directly obtain the position of the defect. The oscillation amplitude will be maximal at the border of the dead region, since the current density will be highest in the direction of the group velocity at the inflection line. In analogy to a hurricane the "eye" is surrounded by a ring of intense currents. Such rings of high amplitude oscillations have already been reported very recently in experiments on $\mathrm{Ag}$ and $\mathrm{Cu}(111)$ surfaces. ${ }^{17}$
For our model FS, along this border the oscillating part of the conductance is, apart from a phase factor, described by

$$
\begin{aligned}
\Delta G(V) \propto & \sin \left(\frac { 4 z _ { 0 } } { b \hbar } \left(\frac{\sqrt{p_{\perp \max } p_{\perp \text { min }}}}{p_{\perp \max }-p_{\perp \min }}\right.\right. \\
& \left.\left.+\arcsin \sqrt{\frac{p_{\perp \max }^{2}-p_{\perp \max } p_{\perp \min }}{2 m \varepsilon_{1}}}\right)\left.\right|_{\varepsilon=\varepsilon_{F}+e V}\right),
\end{aligned}
$$

where $p_{\perp \max }$ and $p_{\perp \min }$ are the maximal and minimal radii of the surface of constant energy in the direction perpendicular to the axis of the cylinder (57), $\varepsilon_{1}$ is the amplitude of corrugation of the FS, and $2 \pi / b$ is the size of the Brillouin zone. Again we find that the depth of the defect is determining the oscillation period, so that for given FS parameters this information can be exploited to investigate the structure of the metal below the surface.

If the open direction is parallel with the surface the highest amplitude will be found with the STM tip straight above the defect. For $\boldsymbol{\rho}_{0}=0$ the conductance oscillations are described by Eq. (73). Clearly, the oscillation pattern is more complicated than that from Eq. (74), since there are contributions from the belly as well as from the neck parts of the FS, plus a sum frequency. For small necks the signal will be dominated by the oscillation due to the belly.

Although the two models presented in this paper are still rather artificial, they provide insights that are quite valuable for experimental work in this field. The most prominent conclusion is that the regular oscillations due to convex parts of the FS, that behave as for the isotropic FS discussed previously, ${ }^{8}$ will often be dominated by signals due to special directions. On any surface that features regions of zero curvature, the strongest conductance fluctuations will come from electrons traveling with the group velocity of that region. Not only does this hold for the inflection lines around the necks in the (111) direction of, e.g., $\mathrm{Cu}, \mathrm{Ag}$ or $\mathrm{Au}$, it is also true for the almost flat facets in the (110) direction of the same metals. In the case of an inflection line the signal will decay as $r^{-5 / 3}$, whereas for a flat facet the signal does not decay at all. This effect can be exploited for imaging defects up to much larger depths than previously estimated. The particular shape of the FS for nearly all metals contain many detailed features that will allow us to check the validity of the conclusions drawn from the measured data. ${ }^{17}$

\section{ACKNOWLEDGMENTS}

The authors thank M. Wenderoth for communicating his unpublished results. One of the authors (Ye.S.A.) is supported by a grant of the European INTAS Young Scientists program (Grant No. 04-83-3750) and one of the authors (Yu.A.K.) was supported by the European Erasmus Mundus program on Nanoscience. This research was supported partly by the program "Nanosystems, nanomaterials, and nanotechnology" of National Academy of Sciences of Ukraine. 
${ }^{1}$ B. Ludoph, M. H. Devoret, D. Esteve, C. Urbina, and J. M. van Ruitenbeek, Phys. Rev. Lett. 82, 1530 (1999).

${ }^{2}$ C. Untiedt, G. R. Bollinger, S. Vieira, and N. Agraït, Phys. Rev. B 62, 9962 (2000).

${ }^{3}$ B. Ludoph and J. M. van Ruitenbeek, Phys. Rev. B 61, 2273 (2000).

${ }^{4}$ A. Halbritter, Sz. Csonka, G. Mihály, O. I. Shklyarevskii, S. Speller, and H. van Kempen, Phys. Rev. B 69, 121411(R) (2004).

${ }^{5}$ A. Namiranian, Yu. A. Kolesnichenko, and A. N. Omelyanchouk, Phys. Rev. B 61, 16796 (2000).

${ }^{6}$ Ye. S. Avotina and Yu. A. Kolesnichenko, Fiz. Nizk. Temp. 30, 209 (2004) [J. Low Temp. Phys. 30, 153 (2004)].

${ }^{7}$ Ye. S. Avotina, A. Namiranian, and Yu. A. Kolesnichenko, Phys. Rev. B 70, 075308 (2004).

${ }^{8}$ Ye. S. Avotina, Yu. A. Kolesnichenko, A. N. Omelyanchouk, A. F. Otte, and J. M. Van Ruitenbeek, Phys. Rev. B 71, 115430 (2005).
${ }^{9}$ I. M. Lifshits, M. Ya. Azbel', and M. I. Kaganov, Electron Theory of Metals (Consultants Bureau, New York, (1973).

${ }^{10}$ A. M. Kosevich, Fiz. Nizk. Temp. 11, 1106 (1985) [Sov. J. Low Temp. Phys. 11, 611 (1985)].

${ }^{11}$ V. V. Ustinov and D. Z. Khusainov, Phys. Status Solidi B 134, 313 (1986).

${ }^{12}$ I. O. Kulik, Yu. N. Mitsai, and A. N. Omelyanchouk, Zh. Eksp. Teor. Fiz. 63, 1051 (1974).

${ }^{13}$ M. Ya. Azbel, Phys. Rev. B 43, 2435 (1991).

${ }^{14}$ I. F. Itskovich and R. I. Shekhter, Fiz. Nizk. Temp. 11, 373 (1985) [Sov. J. Low Temp. Phys. 11, 202 (1985)].

${ }^{15}$ V. P. Maslov and M. V. Fedoriuk, Semi-classical Approximation in Quantum Mechanics (D. Reidel, Dordrecht, 1981).

${ }^{16}$ G. Korn and T. Korn, Mathematical Handbook (McGraw-Hill, New York (1968).

${ }^{17}$ A. Weismann, M. Wenderoth, N. Quaas, and R. G. Ulbrich (unpublished). 